\title{
Correction to: Biomechanical properties of 3D-printed bone scaffolds are improved by treatment with CRFP
}

Carlos G. Helguero ${ }^{1,4}$, Vamiq M. Mustahsan', Sunjit Parmar ${ }^{3}$, Sahana Pentyala ${ }^{3}$, John L. Pfail ${ }^{3}$, Imin Kao ${ }^{1}$, David E. Komatsu ${ }^{2}$ and Srinivas Pentyala ${ }^{3^{*}}$

\section{Correction to: J Orthop Surg Res (2017) 12: 195. https://doi.org/10.1186/s13018-017-0700-2}

In the original publication of this article [1] there was an error in one of the author names. In this publication the correct and incorrect name are indicated.

Originally the author name has been published as:

- John P. Pfail

The correct name is as followed:

- John L. Pfail

The original publication has been corrected.

\begin{abstract}
Author details
'Department of Mechanical Engineering, Stony Brook University, Stony Brook, NY, USA. ${ }^{2}$ Department of Orthopedics, Stony Brook Medical Center, Stony Brook, NY, USA. ${ }^{3}$ Department of Anesthesiology, Stony Brook Medical Center, Stony Brook, NY, USA. ${ }^{4}$ Facultad de Ingeniería en Mecánica y Ciencias de la Producción, Escuela Superior Politécnica del Litoral, ESPOL, Guayaquil,

Ecuador.
\end{abstract}

Received: 7 February 2018 Accepted: 7 February 2018

Published online: 19 February 2018

\section{Reference}

1. Helguero CG, Mustahsan VM, Parmar S, et al. Biomechanical properties of 3D-printed bone scaffolds are improved by treatment with CRFP. J Orthop Surg Res. 2017;12:195. https://doi.org/10.1186/s13018-017-0700-2

\footnotetext{
* Correspondence: Srinivas.pentyala@stonybrook.edu

${ }^{3}$ Department of Anesthesiology, Stony Brook Medical Center, Stony Brook, NY, USA
} 